\title{
Research Article \\ Periodontal Disease and Tooth Loss Are Associated with Lung Cancer Risk
}

\author{
You Chen, ${ }^{1}$ Bao-ling Zhu, ${ }^{2}$ Cong-cong Wu, ${ }^{2}$ Rui-fang Lin, ${ }^{2}$ and Xi Zhang $\mathbb{D}^{2}$ \\ ${ }^{1}$ College of Stomatology, Dalian Medical University, 9 West Section, Lvshun South Road, Lvshunkou District, \\ Dalian City 116044, China \\ ${ }^{2}$ Department of Chemotherapy and Radiotherapy, The Second Affiliated Hospital and Yuying Children's Hospital of Wenzhou \\ Medical University, 109 Xueyuan West Road, Lucheng District, Wenzhou City 325027, China
}

Correspondence should be addressed to Xi Zhang; zhangxiwmu@163.com

Received 9 March 2020; Revised 22 June 2020; Accepted 13 July 2020; Published 27 July 2020

Academic Editor: Noriyoshi Sawabata

Copyright (c) 2020 You Chen et al. This is an open access article distributed under the Creative Commons Attribution License, which permits unrestricted use, distribution, and reproduction in any medium, provided the original work is properly cited.

Background. The associations between periodontal disease, tooth loss, and lung cancer risk remain debatable. Therefore, the purpose of the present study is to evaluate whether periodontal disease and tooth loss are associated with lung cancer risk. Methods. A literature search was performed for relevant studies using PubMed and Embase databases. Risk ratio (RR) with 95\% confidence interval (CI) was applied as effect size to summarize the associations between periodontal disease, tooth loss, and lung cancer risk. A further dose-response analysis was also performed. Results. A total of twelve studies comprising 263,238 participants were included. The results indicated that periodontal disease was positively associated with lung cancer risk $(\mathrm{RR}=1.37,95 \% \mathrm{CI}=1.16-1.63)$. There was a positive association between tooth loss and lung cancer risk $(\mathrm{RR}=1.69,95 \% \mathrm{CI}=$ 1.46-1.96). Moreover, there was a significantly linear dose-response relationship between tooth loss and lung cancer risk, and every 5 increment in tooth loss was associated with $10 \%$ increased lung cancer risk. Similar results were obtained in subgroup analysis. Conclusions. Periodontal disease and tooth loss are increased risk factors for lung cancer. Prevention and treatment of periodontal disease may be effective potential prevention strategies for lung cancer.

\section{Introduction}

Worldwide, lung cancer is the most frequently diagnosed cancer and the leading cause of cancer-related death, with approximately 2.1 million new lung cancer cases and 1.8 million deaths [1]. Surgical resection is the best radical treatment for lung cancer. However, most patients have lost the chance of radical resection at the initial diagnosis and are usually treated with palliative chemotherapy and/or radiotherapy. In spite of the improvement of the treatment level, the prognosis of lung cancer patients is still poor, with a low survival rate [2]. Therefore, early and effective prevention strategies for lung cancer risk are quite significant.

Periodontal disease is a chronic inflammatory disease of the periodontium caused by periodontal pathogen infection, resulting in the damage of tooth-supporting tissues and finally leading to tooth loss [3]. Severe periodontitis is the sixth-most prevalent public health condition worldwide, which has affected approximately $10.8 \%$ of the total population [4]. As a chronic inflammatory disease, several studies have reported that periodontal disease has been demonstrated to increase the risk of several malignancies such as head and neck cancer [5], oral cancer [6,7], digestive tract cancer $[8,9]$, pancreatic cancer $[10]$, and prostate cancer [11]. Unfortunately, there are no consistent conclusions on the associations between periodontal disease, tooth loss, and lung cancer risk because some studies show positive associations, while others show null associations.

Therefore, the purpose of our meta-analysis is to evaluate whether periodontal disease and tooth loss are associated 
with lung cancer risk and whether prevention of periodontal disease is an effective potential prevention strategy for lung cancer.

\section{Material and Methods}

2.1. Literature Search. A systematic literature search was performed for the relevant studies on associations between periodontal disease, tooth loss, and lung cancer risk using PubMed and Embase databases (up to September 2019). The search strategy was as follows: (periodontitis OR peridentitis OR "periodontal disease" OR "periodontal diseases" OR parodontopathy OR "gingival disease" OR "teeth number" OR "oral health" OR "dental health" OR "periodontal attachment loss" OR "periodontal pocket” OR “alveolar bone loss” OR gingivitis OR edentulous OR "tooth loss" OR "teeth loss" OR "dental plaque" OR edentulism) AND (lung cancer OR lung carcinoma OR lung tumor OR lung neoplasm). Furthermore, we manually searched the references of reviews and relevant studies to identify other eligible studies.

2.2. Eligibility Criteria. Included studies must meet the following eligibility criteria: (1) the exposure factor was periodontal disease and/or tooth loss; (2) the endpoint outcome was lung cancer risk; (3) the effect size of outcome was hazard ratio (HR), odds ratio (OR), or risk ratio (RR) with $95 \%$ confidence interval (CI); (4) the study type was cohort study or casecontrol study and the full text was available. In addition, only the most comprehensive study was included for meta-analysis if there were several duplicated studies. Some data reported only in the excluded duplicated studies were extracted into the included duplicated study for further analysis.

2.3. Data Extraction and Quality Assessment. Two reviewers independently screened literatures and extracted data as follows: first author, study type, study name, publication country and year, follow-up time, age, type of exposure ascertainment, type of lung cancer ascertainment, sample size, number of cases, exposure type, effect size with 95\% CI, and controlled confounding factors. Moreover, if the same study provided several risk estimates and these risk estimates had major gaps for confounder control, only the risk estimates with the greatest control for confounding factors were extracted for metaanalysis. The study quality of the included studies was assessed by the Newcastle-Ottawa Scale (NOS) [12]. Any differences on literature selection, data extraction, and quality assessment were resolved by discussion.

2.4. Statistical Analysis. RR with $95 \%$ CI was used as effect size to summarize the associations between periodontal disease, tooth loss, and lung cancer risk. OR value provided by case-control study could be almost equal to the RR because the lung cancer incidence was extremely low, and thus, OR was used as RR in the data synthesis [13]. A pooled risk estimate was synthesized for further meta-analysis when there were multiple risk estimates based on different subpopulations in one study. For the meta-analysis of tooth loss, we utilized the risk estimate which represented the most severe degree of tooth loss since there was no uniform standard for tooth loss [10].
To assess the impact of the number of tooth loss on lung cancer risk, we also performed a dose-response analysis which needed the assigned values of tooth loss, distributions of cases and noncases, and risk estimates in each category [14]. A midpoint of the interval of the tooth loss was chosen as assigned value for the risk estimate in each category. For the open-ended interval [15], we assumed that the range was the same as that of the adjacent interval. Restricted cubic splines were utilized to test a nonlinear dose-response relationship, and generalized least-squares regressions were used to test a linear dose-response relationship $[16,17]$.

The Cochran $Q$ test and the $I^{2}$ statistic were performed to evaluate heterogeneity among studies, and the definition of statistically significant heterogeneity was $p<0.10$ and/or $I^{2}$ $>50 \%$ [18]. A random-effect model was used when the heterogeneity was significant, and on the contrary, a fixed-effect model was chosen. The Galbraith plot was used to explore which study contributed substantial heterogeneity. The overall analysis was performed by including all studies. Subgroup analysis was performed stratified by study type, study quality, cancer ascertainment, exposure ascertainment, sample size, and country. Moreover, adequate control of confounding factors was essential to obtain valid results and to reduce misleading results, and thus, subgroup analysis based on different controlled confounding factors was conducted. Moreover, we further performed in-depth subgroup analysis not only to control for smoking but also to control for smoking amount and duration because smoking was an extremely important risk factor for lung cancer [19]. Publication bias was evaluated by Egger's and Begg's tests [20, 21]. In addition, a trim-and-fill analysis was conducted to evaluate the impact of publication bias on the results when publication bias existed [22].

All statistical analyses were conducted in Stata software version 12.0 (Stata Corporation, USA). A two-sided $p<$ 0.05 was considered statistically significant.

\section{Results}

3.1. Selection of Studies. There were 1228 studies initially obtained from the literature search, among which 662 studies were from PubMed database and 566 studies were from Embase database. 1185 studies were excluded according to the title and abstract, and the remaining 43 studies were needed to review the full texts. After reviewing these full texts, 31 studies were excluded because these studies did not meet the eligibility criteria, and 12 studies were included for our meta-analysis [23-34]. The study selection process and the reasons for exclusion were shown in Figure 1.

3.2. Study Characteristics. The twelve studies were published from 2003 to 2019. Among these studies, five studies were from the USA, two studies were from Japan, and one study was from Turkey, Finland, Greece, Australia, and UK, respectively. In the type of study design, nine studies were cohort studies [24-28, 30, 31, 33, 34] and three studies were case-control studies $[23,29,32]$. In terms of exposure ascertainment, there were six studies using clinical periodontal examination $[24,26,27,29,33,34]$, and another six studies 


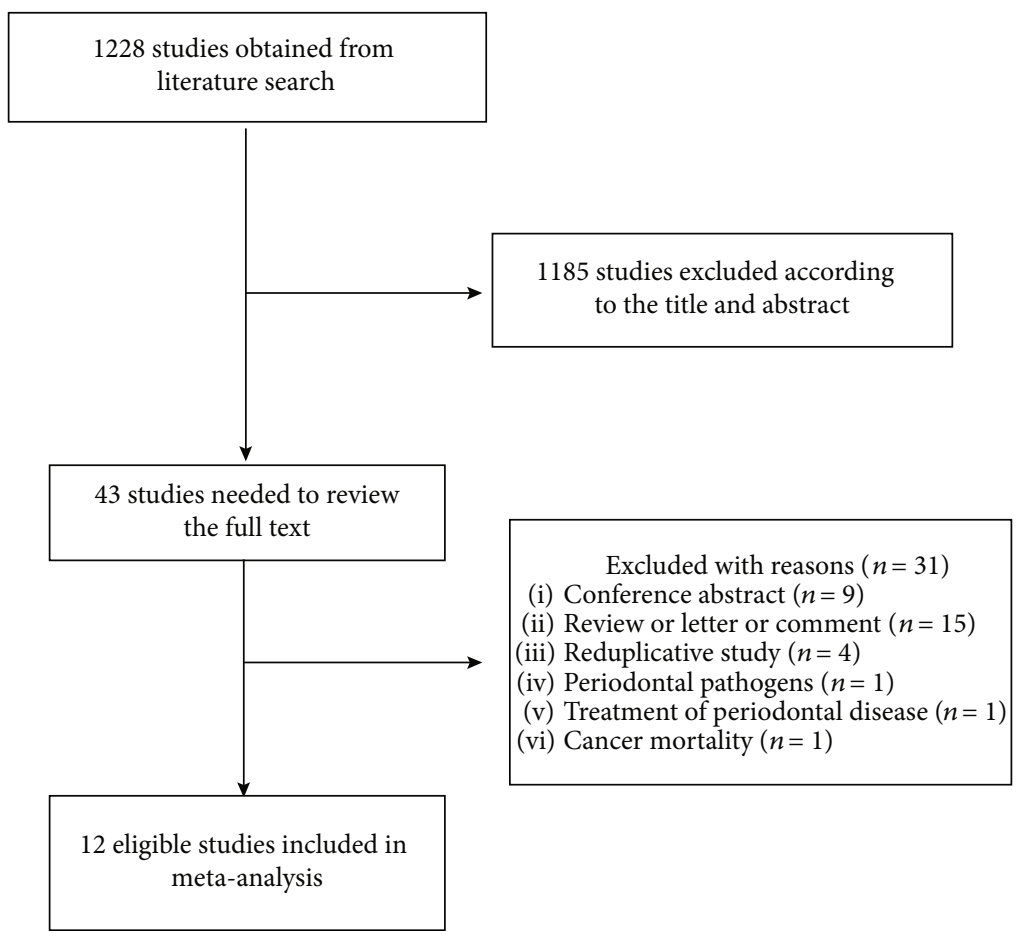

Figure 1: Process of literature search and study selection.

were self-reported measures [23, 25, 28, 30-32]. For the exposure factors, five studies only assessed periodontal disease [24, 27-30], three studies only assessed tooth loss [25, $32,33]$, and four studies assessed both periodontal disease and tooth loss $[23,26,31,34]$. For the control for confounding factors, age and sex were available in ten studies, smoking in eleven studies, alcohol drinking in eleven studies, BMI in seven studies, and diabetes in five studies. Moreover, among the included studies which have controlled for smoking, six and two studies further controlled for smoking amount and smoking duration, respectively. The main baseline characteristics of the included studies were shown in Table 1.

3.3. The Association between Periodontal Disease and Lung Cancer Risk. There were nine studies evaluating the association between periodontal disease and lung cancer risk $[23,24,26-31,34]$. A random-effect model was used to pool RR due to a significant heterogeneity $\left(I^{2}=62.7 \%\right)$, and the result indicated a positive association between periodontal disease and lung cancer risk $(\mathrm{RR}=1.37,95 \% \mathrm{CI}=1.16$ 1.63, Figure 2). The Galbraith plot showed that the study by Guven et al. contributed relatively substantial heterogeneity, and thus, we recalculated the pooled RR after excluding the study by Guven et al. [24]. The pooled RR with 95\% CI indicated a consistent result, without significant heterogeneity $\left(\mathrm{RR}=1.43,95 \% \mathrm{CI}=1.30-1.56, I^{2}=7.3 \%\right.$, Figure 2$)$. After excluding case-control studies, subgroup analysis based on cohort study also showed that periodontal disease could increase lung cancer risk $(\mathrm{RR}=1.33,95 \% \mathrm{CI}=1.09-1.62)$. Subgroup analysis controlling for smoking indicated a positive relationship between periodontal disease and lung cancer risk $(\mathrm{RR}=1.44,95 \% \mathrm{CI}=1.31-1.58)$, and similar results were also obtained after further controlling for smoking amount
$(\mathrm{RR}=1.40,95 \% \mathrm{CI}=1.27-1.54)$ and duration $(\mathrm{RR}=1.86$, $95 \% \mathrm{CI}=1.40-2.48)$. The pooled $\mathrm{RR}$ with control for all potential important confounding factors (including age, sex, smoking, alcohol drinking, BMI, and diabetes) was 1.54 (95\% CI $=1.34-1.78)$, suggesting that periodontal disease was a strong factor for lung cancer risk (Table 2).

Moreover, similar results were acquired in the subgroup analysis after dividing into groups by gender, publication country, study quality, sample size, cancer ascertainment, exposure ascertainment, and various controlled confounding factors, indicating that the periodontal disease was positively associated with lung cancer risk (Table 2).

3.4. The Association between Tooth Loss and Lung Cancer Risk. There were seven studies estimating the association between tooth loss and lung cancer risk [23, 25, 26, 31-34]. The result indicated that there was a positive association between tooth loss and lung cancer risk $(\mathrm{RR}=1.69,95 \%$ $\mathrm{CI}=1.46-1.96$, Figure 3 ), without significant heterogeneity $\left(I^{2}=0.0 \%\right)$. In terms of study design, subgroup analysis stratified by cohort study $(\mathrm{RR}=1.73,95 \% \mathrm{CI}=1.46-2.05)$ and case-control study $(\mathrm{RR}=1.58,95 \% \mathrm{CI}=1.16-2.14)$ also showed a positive association. Subgroup analysis based on the control for smoking status, amount, and duration obtained similar results, and the pooled RR was 1.80 after controlling for all potential important confounding factors (including age, sex, smoking, alcohol drinking, BMI, and diabetes) (Table 2). Moreover, for the subgroup analysis after dividing into groups by publication country, study quality, gender, sample size, cancer ascertainment, exposure ascertainment, and various controlled confounding factors, we obtained similar results which indicated a positive relationship between tooth loss and lung cancer risk (Table 2). 


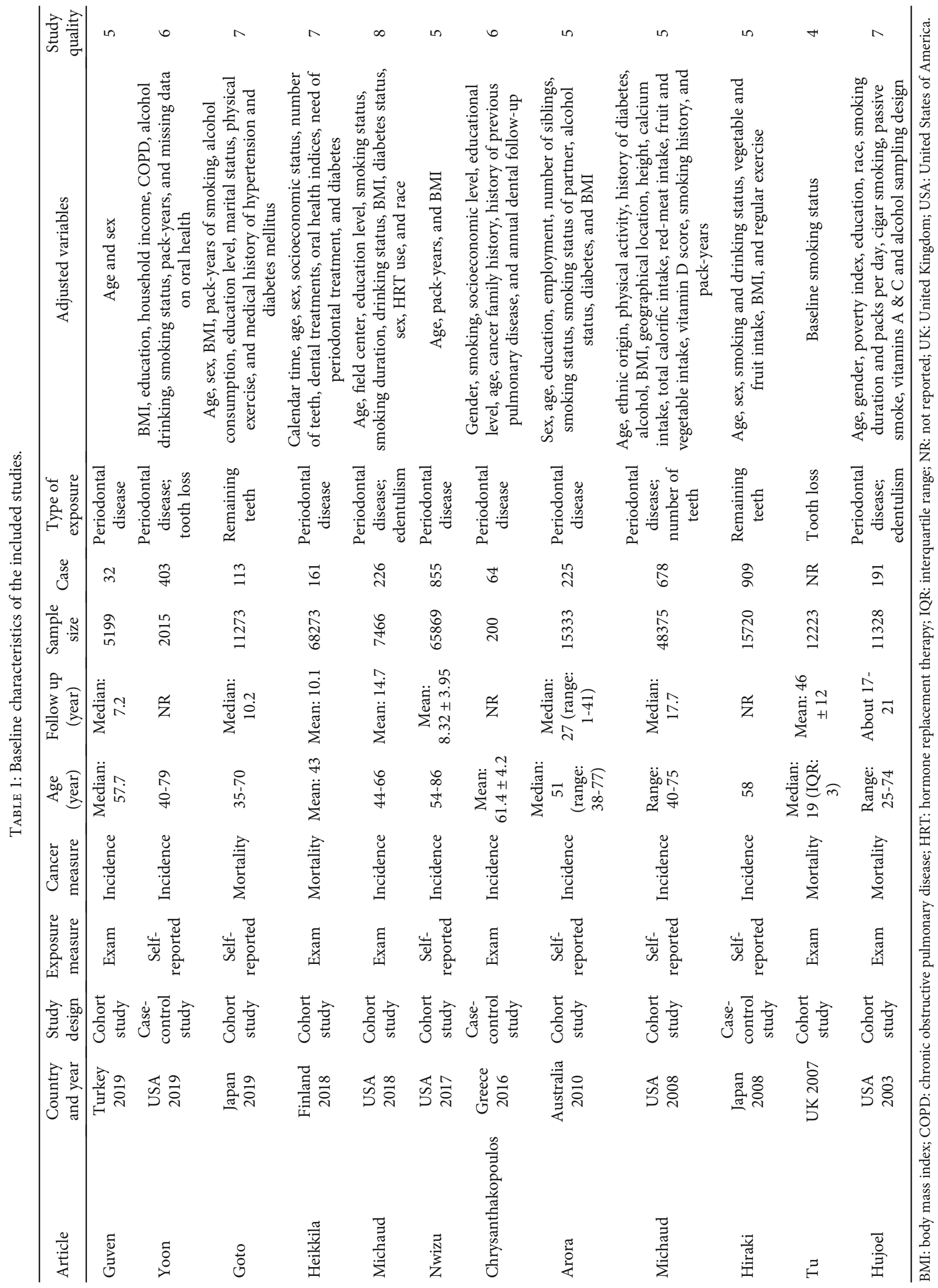




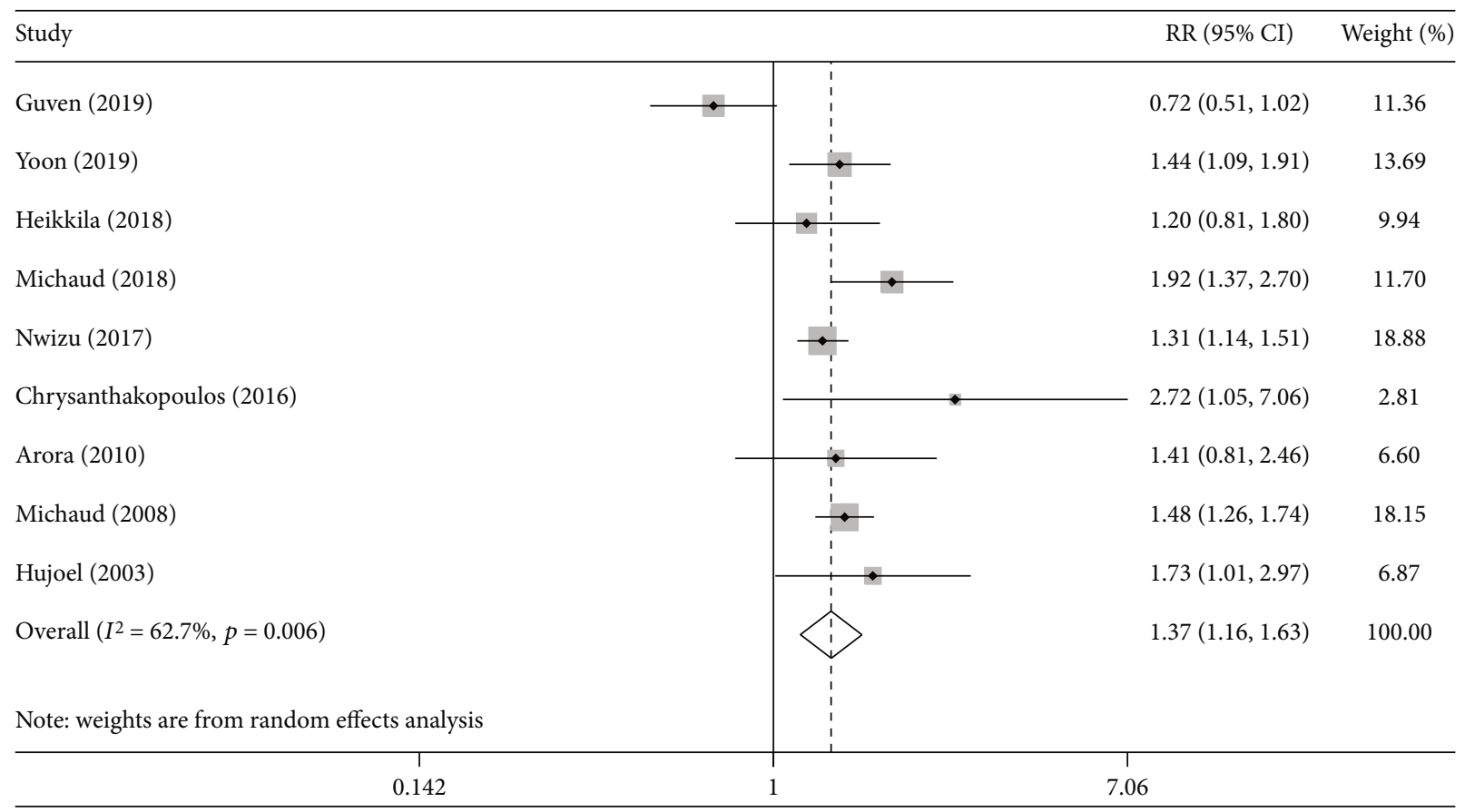

(a)

\begin{tabular}{|c|c|c|c|}
\hline Study & & $\mathrm{RR}(95 \% \mathrm{CI})$ & Weight (\%) \\
\hline Yoon (2019) & $\stackrel{i}{\circ}$ & $1.44(1.09,1.91)$ & 10.21 \\
\hline Heikkila (2018) & $\rightarrow !$ & $1.20(0.81,1.80)$ & 5.04 \\
\hline Michaud (2018) & $\stackrel{1}{1}$ & $1.92(1.37,2.70)$ & 6.98 \\
\hline Nwizu (2017) & $\rightarrow$ & $1.31(1.14,1.51)$ & 40.67 \\
\hline Chrysanthakopoulos (2016) & : & $2.72(1.05,7.06)$ & 0.89 \\
\hline Arora (2010) & $\rightarrow$ & $1.41(0.81,2.46)$ & 2.60 \\
\hline Michaud (2008) & $\rightarrow$ & $1.48(1.26,1.74)$ & 30.84 \\
\hline Hujoel (2003) & $\stackrel{1}{1}$ & $1.73(1.01,2.97)$ & 2.76 \\
\hline Overall $\left(I^{2}=7.3 \%, p=0.374\right)$ & 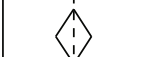 & $1.43(1.30,1.56)$ & 100.00 \\
\hline $\begin{array}{c}1 \\
0.142\end{array}$ & 1 & & \\
\hline
\end{tabular}

(b)

FIGURE 2: Result of the association between periodontal disease and lung cancer risk: (a) all studies; (b) excluding the study by Guven et al.

In addition, we performed a dose-response analysis to explore the impact of the number of tooth loss on lung cancer risk, and the result indicated that there was no nonlinear relationship ( $p$ for nonlinearity $=0.96$ ). Indeed, a significant linear dose-response relationship was confirmed by the generalized least-squares regressions ( $p$ for linearity $<0.01$, Figure 4 ). Every 5 increment in tooth loss was associated with $10 \%$ increased lung cancer risk $(\mathrm{RR}=1.10,95 \% \mathrm{CI}=1.04-1.17)$.

\section{Discussion}

Lung cancer is the cancer of the highest morbidity and mortality worldwide which still have a poor prognosis even after effective treatment $[1,2]$. Thus, it is urgent to find its risk factors for effective prevention. Recent studies have confirmed that periodontal disease and tooth loss are associated with several solid tumors such as oral cancer [6,7], head and neck cancer [5], and pancreatic cancer [10]. However, the 
TABLE 2: The results for the associations between periodontal disease, tooth loss, and lung cancer risk.

\begin{tabular}{|c|c|c|c|c|c|}
\hline & $N$ & $\mathrm{RR}$ & $p_{\mathrm{RR}}$ & Heter & Publication bias \\
\hline \multicolumn{6}{|l|}{ Periodontal disease } \\
\hline Overall & 9 & $1.37(1.16-1.63)$ & $<0.001$ & $62.70 \%$ & Begg's test $=0.602$; Egger' $\mathrm{s}$ test $=0.771$ \\
\hline Overall without Guven & 8 & $1.43(1.30-1.56)$ & $<0.001$ & $7.30 \%$ & Begg's test $=0.386$; Egger' s test $=0.168$ \\
\hline \multicolumn{6}{|l|}{ Study type } \\
\hline Cohort & 7 & $1.33(1.09-1.62)$ & 0.004 & $68.80 \%$ & Begg's test $=1.000$; Egger s test $=0.880$ \\
\hline Cohort without Guven & 6 & $1.42(1.29-1.56)$ & $<0.001$ & $13.10 \%$ & Begg's test $=0.707 ;$ Egger' s test $=0.460$ \\
\hline Case-control study & 2 & $1.52(1.16-1.98)$ & 0.002 & $37.00 \%$ & Begg's test $=1.000 ;$ Egger's test $=/$ \\
\hline \multicolumn{6}{|l|}{ Cancer ascertainment } \\
\hline Cancer incidence & 7 & $1.37(1.12-1.68)$ & 0.002 & $70.40 \%$ & Begg's test $=0.764 ;$ Egger' $s$ test $=0.834$ \\
\hline Cancer incidence without Guven & 6 & $1.43(1.30-1.57)$ & $<0.001$ & $21.00 \%$ & Begg's test $=0.452$; Egger's test $=0.127$ \\
\hline Cancer mortality & 3 & $1.22(1.02-1.45)$ & 0.027 & $0.00 \%$ & Begg's test $=0.296 ;$ Egger' $\mathrm{s}$ test $=0.374$ \\
\hline \multicolumn{6}{|l|}{ Exposure ascertainment } \\
\hline Exam & 5 & $1.41(0.90-2.21)$ & 0.135 & $79.70 \%$ & Begg' s test $=0.806 ;$ Egger's test $=0.493$ \\
\hline Exam without Guven & 4 & $1.65(1.32-2.07)$ & $<0.001$ & $30.30 \%$ & Begg's test $=1.000$; Egger' s test $=0.637$ \\
\hline Self-reported & 4 & $1.39(1.26-1.53)$ & $<0.001$ & $0.00 \%$ & Begg's test $=1.000 ;$ Egger' s test $=0.719$ \\
\hline \multicolumn{6}{|l|}{ Sample size } \\
\hline$<12000$ & 5 & $1.46(0.96-2.21)$ & 0.075 & $79.70 \%$ & Begg's test $=0.806 ;$ Egger' $s$ test $=0.613$ \\
\hline$<12000$ without Guven & 4 & $1.67(1.37-2.03)$ & $<0.001$ & $0.00 \%$ & Begg's test $=0.308 ;$ Egger's test $=0.278$ \\
\hline$\geq 12000$ & 4 & $1.37(1.24-1.51)$ & $<0.001$ & $0.00 \%$ & Begg's test $=0.734 ;$ Egger's test $=0.816$ \\
\hline \multicolumn{6}{|l|}{ Country } \\
\hline Not Asia & 8 & $1.43(1.30-1.56)$ & $<0.001$ & $7.30 \%$ & Begg's test $=0.386 ;$ Egger's test $=0.168$ \\
\hline \multicolumn{6}{|l|}{ Sex } \\
\hline Male & 3 & $1.19(0.65-2.17)$ & 0.575 & $88.80 \%$ & Begg's test $=1.000$; Egger's test $=0.717$ \\
\hline Female & 3 & $1.34(1.17-1.52)$ & $<0.001$ & $0.00 \%$ & Begg's test $=1.000 ;$ Egger's test $=0.772$ \\
\hline \multicolumn{6}{|l|}{ Study quality } \\
\hline$\geq 7$ & 3 & $1.60(1.27-2.03)$ & $<0.001$ & $37.2 \%$ & Begg's test $=1.000 ;$ Egger' $s$ test $=0.871$ \\
\hline$<7$ & 6 & $1.30(1.05-1.59)$ & 0.014 & $69.1 \%$ & Begg's test $=1.000$; Egger' $s$ test $=0.927$ \\
\hline \multicolumn{6}{|l|}{ Adjusted variables } \\
\hline Sex+age & 8 & $1.37(1.12-1.67)$ & 0.002 & $67.10 \%$ & Begg's test $=0.536$; Egger's test $=0.807$ \\
\hline Smoking & 7 & $1.44(1.31-1.58)$ & $<0.001$ & $11.60 \%$ & Begg's test $=0.368 ;$ Egger' s test $=0.072$ \\
\hline Alcohol drinking & 5 & $1.53(1.36-1.73)$ & $<0.001$ & $0.00 \%$ & Begg's test $=0.462 ;$ Egger's test $=0.506$ \\
\hline BMI & 5 & $1.42(1.30-1.56)$ & $<0.001$ & $12.3 \%$ & Begg's test $=0.806 ;$ Egger's test $=0.346$ \\
\hline Diabetes & 4 & $1.50(1.31-1.71)$ & $<0.001$ & $9.3 \%$ & Begg's test $=1.000 ;$ Egger's test $=0.961$ \\
\hline Smoking+alcohol drinking & 5 & $1.53(1.36-1.73)$ & $<0.001$ & $0.00 \%$ & Begg's test $=0.462$; Egger's test $=0.506$ \\
\hline Smoking+alcohol drinking+sex+age & 4 & $1.55(1.36-1.78)$ & $<0.001$ & $0.00 \%$ & Begg's test $=1.000 ;$ Egger's test $=0.530$ \\
\hline Smoking+alcohol drinking + sex + age $+\mathrm{BMI}+$ diabetes & 3 & $1.54(1.34-1.78)$ & $<0.001$ & $0.0 \%$ & Begg's test $=1.000 ;$ Egger' $\mathrm{s}$ test $=0.741$ \\
\hline \multicolumn{6}{|l|}{ Adjusted smoking factor } \\
\hline Amount of smoking & 5 & $1.40(1.27-1.54)$ & $<0.001$ & $0.0 \%$ & Begg' s test $=1.000 ;$ Egger's $s$ test $=0.354$ \\
\hline Duration of smoking & 2 & $1.86(1.40-2.48)$ & $<0.001$ & $0.0 \%$ & Begg's test $=1.000 ;$ Egger's test $=/$ \\
\hline \multicolumn{6}{|l|}{ Tooth loss } \\
\hline Overall & 7 & $1.69(1.46-1.96)$ & $<0.001$ & $0.00 \%$ & Begg's test $=0.368 ;$ Egger's test $=0.868$ \\
\hline \multicolumn{6}{|l|}{ Study type } \\
\hline Cohort & 5 & $1.73(1.46-2.05)$ & $<0.001$ & $0.00 \%$ & Begg's test $=0.462 ;$ Egger's test $=0.956$ \\
\hline Case-control study & 2 & $1.58(1.16-2.14)$ & 0.003 & $0.00 \%$ & Begg' $s$ test $=1.000 ;$ Egger's test $=/$ \\
\hline
\end{tabular}


TABLE 2: Continued.

\begin{tabular}{|c|c|c|c|c|c|}
\hline & $N$ & $\mathrm{RR}$ & $p_{\mathrm{RR}}$ & Heter & Publication bias \\
\hline \multicolumn{6}{|l|}{ Cancer ascertainment } \\
\hline Cancer incidence & 4 & $1.73(1.47-2.05)$ & $<0.001$ & $0.00 \%$ & Begg' s test $=0.308 ;$ Egger's test $=0.599$ \\
\hline Cancer mortality & 3 & $1.54(1.10-2.14)$ & 0.011 & $0.00 \%$ & Begg's test $=0.296 ;$ Egger' $\mathrm{s}$ test $=0.011$ \\
\hline \multicolumn{6}{|l|}{ Exposure ascertainment } \\
\hline Exam & 3 & $1.80(1.28-2.54)$ & 0.001 & $38.00 \%$ & Begg's test $=0.296 ;$ Egger's test $=0.028$ \\
\hline Self-reported & 4 & $1.67(1.41-1.97)$ & $<0.001$ & $0.00 \%$ & Begg's test $=0.734 ;$ Egger's test $=0.679$ \\
\hline \multicolumn{6}{|l|}{ Sample size } \\
\hline$<12000$ & 4 & $1.82(1.40-2.37)$ & $<0.001$ & $0.00 \%$ & Begg's test $=0.734 ;$ Egger's test $=0.605$ \\
\hline$\geq 12000$ & 3 & $1.63(1.36-1.96)$ & $<0.001$ & $0.00 \%$ & Begg's test $=0.296$; Egger's test $=0.043$ \\
\hline \multicolumn{6}{|l|}{ Country } \\
\hline Asia & 2 & $1.62(1.20-2.19)$ & 0.002 & $0.00 \%$ & Begg's test $=1.000 ;$ Egger's test $=/$ \\
\hline Not Asia & 5 & $1.72(1.45-2.04)$ & $<0.001$ & $0.00 \%$ & Begg's test $=0.221 ;$ Egger' $\mathrm{s}$ test $=0.902$ \\
\hline \multicolumn{6}{|l|}{ Sex } \\
\hline Male & 3 & $1.66(1.15-2.41)$ & 0.007 & $70.40 \%$ & Begg's test $=1.000 ;$ Egger's test $=0.637$ \\
\hline Female & 2 & $1.49(1.02-2.19)$ & 0.040 & $0.00 \%$ & Begg's test $=1.000 ;$ Egger's test $=/$ \\
\hline \multicolumn{6}{|l|}{ Study quality } \\
\hline$\geq 7$ & 3 & $1.90(1.39-2.58)$ & $<0.001$ & $17.2 \%$ & Begg's test $=1.000 ;$ Egger's test $=0.643$ \\
\hline$<7$ & 4 & $1.64(1.38-1.94)$ & $<0.001$ & $0.0 \%$ & Begg's test $=0.308 ;$ Egger's test $=0.140$ \\
\hline \multicolumn{6}{|l|}{ Adjusted variables } \\
\hline Sex+age & 5 & $1.72(1.47-2.02)$ & $<0.001$ & $0.00 \%$ & Begg's test $=1.000$; Egger's test $=0.827$ \\
\hline Smoking & 7 & $1.69(1.46-1.96)$ & $<0.001$ & $0.00 \%$ & Begg's test $=0.368 ;$ Egger $s$ test $=0.868$ \\
\hline Alcohol drinking & 6 & $1.71(1.47-2.00)$ & $<0.001$ & $0.00 \%$ & Begg's test $=1.000 ;$ Egger's test $=0.861$ \\
\hline BMI & 5 & $1.74(1.48-2.03)$ & $<0.001$ & $0.0 \%$ & Begg's test $=0.221 ;$ Egger's test $=0.537$ \\
\hline Diabetes & 3 & $1.80(1.50-2.16)$ & $<0.001$ & $4.7 \%$ & Begg's test $=0.296 ;$ Egger's test $=0.478$ \\
\hline Smoking+alcohol drinking & 6 & $1.71(1.47-2.00)$ & $<0.001$ & $0.00 \%$ & Begg's test $=1.000 ;$ Egger's test $=0.861$ \\
\hline Smoking+alcohol drinking+sex+age & 5 & $1.72(1.47-2.02)$ & $<0.001$ & $0.00 \%$ & Begg's test $=1.000 ;$ Egger's test $=0.827$ \\
\hline Smoking+alcohol drinking+sex+age+BMI+diabetes & 3 & $1.80(1.50-2.16)$ & $<0.001$ & $4.7 \%$ & Begg's test $=0.296$; Egger's test $=0.478$ \\
\hline \multicolumn{6}{|l|}{ Adjusted smoking factor } \\
\hline Amount of smoking & 4 & $1.67(1.40-1.99)$ & $<0.001$ & $0.0 \%$ & Begg's test $=0.089 ;$ Egger' $s$ test $=0.387$ \\
\hline Duration of smoking & 2 & $1.93(1.05-3.57)$ & 0.035 & $55.3 \%$ & Begg's test $=1.000 ;$ Egger's test $=/$ \\
\hline
\end{tabular}

Heter: heterogeneity; BMI: body mass index; $N$ : the number of studies; RR: risk ratio; $p_{\mathrm{RR}}$ : $p$ value for the risk ratio; “”: not applicable because Egger’s test could not be conducted if the study number was only two.

relationships between periodontal disease, tooth loss, and lung cancer risk are still controversial. Thus, we performed a meta-analysis to explore the relationships between periodontal disease, tooth loss, and lung cancer risk.

Twelve eligible studies comprising 263,238 participants were included in this meta-analysis. The results indicated that periodontal disease $(\mathrm{RR}=1.37,95 \% \mathrm{CI}=1.16-1.63)$ and tooth loss $(\mathrm{RR}=1.69,95 \% \mathrm{CI}=1.46-1.96)$ were positively associated with lung cancer risk. Moreover, similar results were obtained in subgroup analysis by study design, publication country, study quality, gender, sample size, cancer ascertainment, exposure ascertainment, and controlled confounding factors. The results of a dose-response analysis showed that there was a significantly linear relationship between tooth loss and lung cancer risk, and the lung cancer risk increased by $10 \%$ for 5 tooth increment in tooth loss, with a monotonically increasing trend.

In exploring the relationship between periodontal disease and lung cancer risk, four studies used self-reported measure to identify periodontal disease. Researchers may be concerned about whether self-reported periodontal disease was validated. Previous several systematic reviews have demonstrated that self-reported periodontal disease had acceptable validity and self-reported measure was feasible for monitoring periodontal disease in epidemiological studies $[35,36]$. Moreover, numerous validation studies have also showed that self-reported measure was valid for the assessment of periodontal disease in different populations [37-40]. Indeed, the results of subgroup analysis based on self-reported periodontal disease showed that there was a positive relationship 


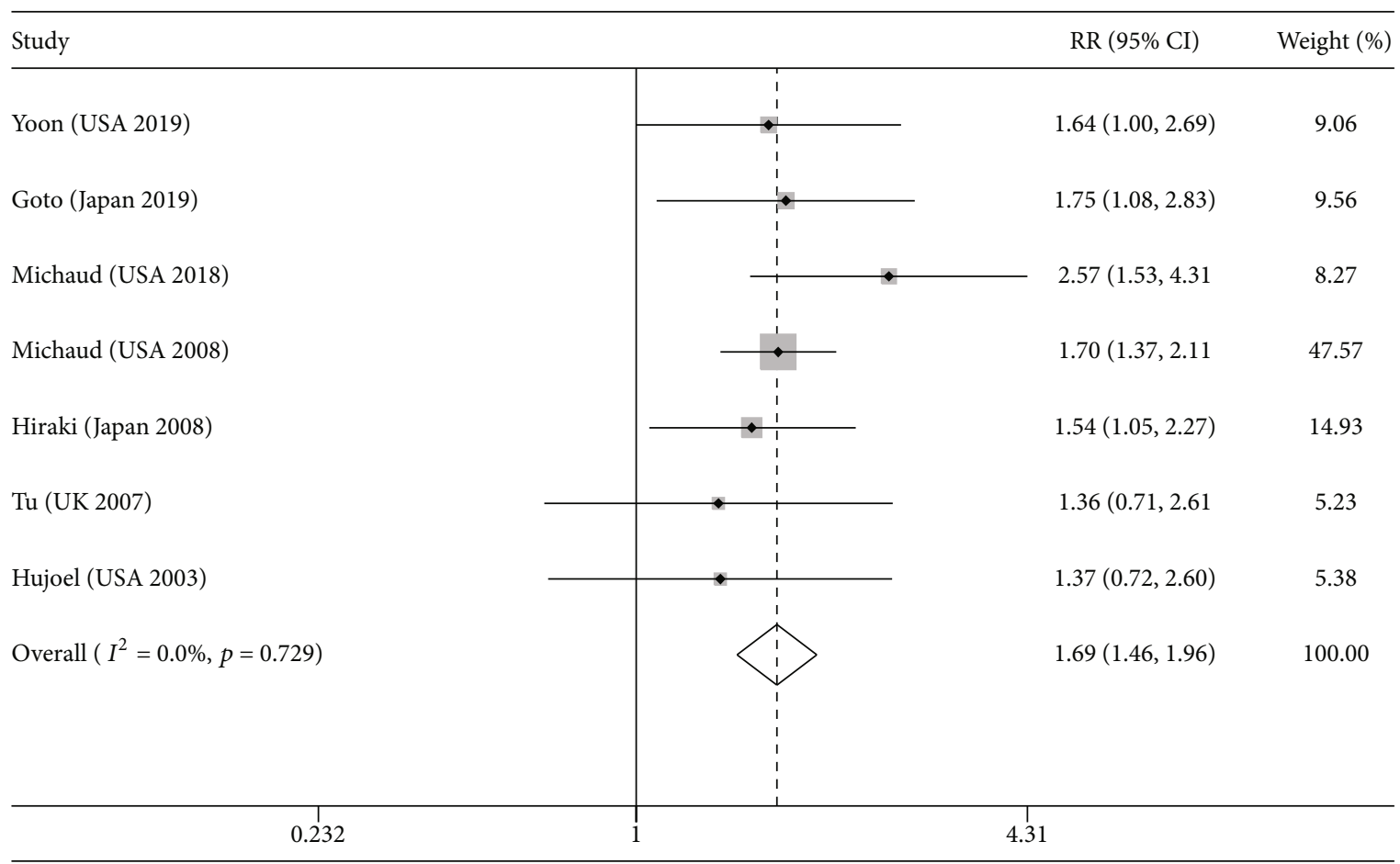

FIgURE 3: Result of the association between tooth loss and lung cancer risk.

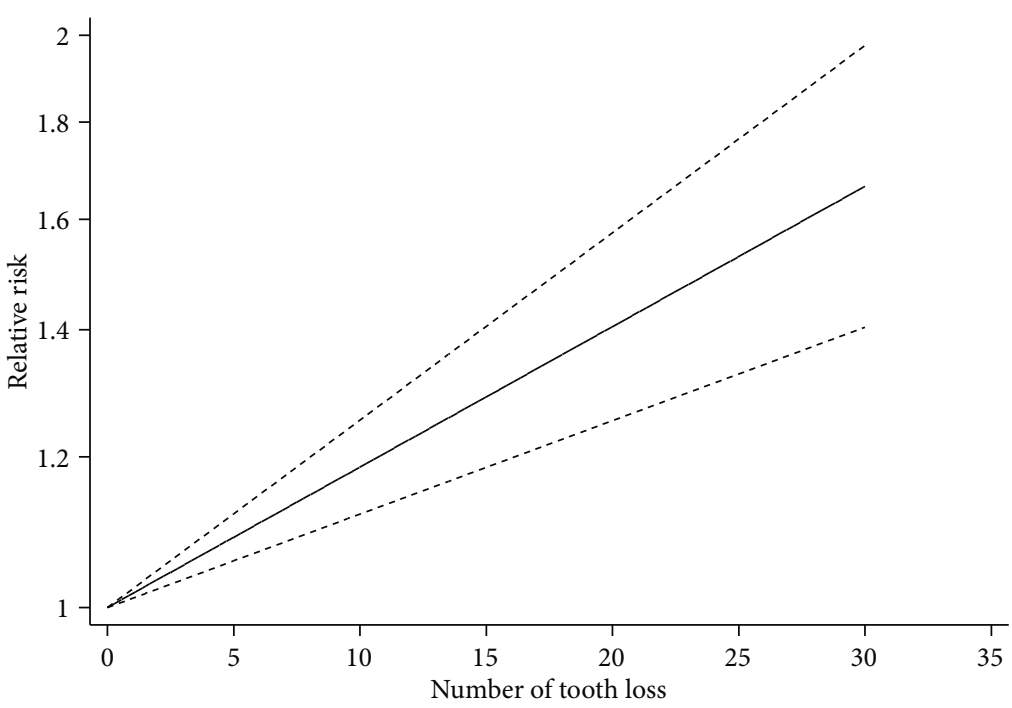

Figure 4: A linear dose-response relationship between tooth loss and lung cancer risk.

between periodontal disease and lung cancer risk $(\mathrm{RR}=1.39$, $95 \% \mathrm{CI}=1.26-1.53$ ), which was consistent with the results of subgroup analysis based on clinical periodontal examination. However, the adequacy for self-reported periodontal disease may depend on the education level and extent of access to routine oral healthcare in the population. Thus, the use of self-reported measure to identify periodontal disease may misclassify periodontal disease and underestimate the status of periodontal disease, which may weaken the association between periodontal disease and lung cancer risk. Future large-scale, well-designed diagnostic studies are needed to explore the validity of self-reported periodontal disease.
Periodontal disease and lung cancer were affected by many common risk factors $[41,42]$. Therefore, the impact of these common confounding factors on the associations between periodontal disease, tooth loss, and lung cancer should be considered. We performed subgroup analyses based on various confounding factors, and the results confirmed the validity of our results. Among these controlled confounding factors, smoking was an extremely important risk factor for both periodontal disease and lung cancer. In order to extensively explore the impact of smoking on our results, it is essential not only to control for smoking but also to control for the smoking amount and duration, and our 
results still showed that there was a positive association between periodontal disease and lung cancer risk. Furthermore, a similar result was obtained after controlling for all potential important confounding factors (including age, sex, smoking, alcohol drinking, BMI, and diabetes), suggesting that periodontal disease was a strong risk factor for lung cancer risk. Future homogeneous, large-scale, and well-designed studies are needed to explore the associations between periodontal disease and lung cancer.

The mechanism of the positive relationship between periodontal disease and lung cancer was unclear. The associations between infection, inflammation, and lung cancer may be the most probable explanation [43]. Emerging evidence has indicated that infections could cause several types of malignant tumors, with approximately 1.2 million cases every year worldwide [44-46]. As a chronic inflammation caused by periodontal pathogen infections, periodontal disease could increase the levels of C-reactive protein, IL-6, IFN- $\gamma$, and IL-1 $\beta$ [47-49]. Periodontal pathogens and inflammation products entered into the bloodstream, which lead to systemic inflammatory response [50,51]. Indeed, several studies have confirmed that high levels of $\mathrm{C}$ reactive protein, IL- 6 , IFN- $\gamma$, and IL- $1 \beta$ were positively associated with lung cancer risk [52-54]. Dental plaque including supragingival plaque and subgingival plaque contained a good deal of bacteria in patients with periodontal disease [55]. Pneumonia caused by aspiration of oral bacteria may be another important mechanism [56-58]. Several studies have reported a positive relationship between pneumonia and lung cancer risk $[59,60]$. Understandably, as a clinical indicator of periodontal disease, tooth loss could reflect the degree of poor oral health and was associated with lung cancer risk, with a linear relationship. Furthermore, further studies are required to explore the underlying mechanisms of the relationships between periodontal disease, tooth loss, and lung cancer risk.

Based on the present status that severe periodontitis and lung cancer were public health problems worldwide and there was a relationship between periodontal disease and lung cancer risk, it was a meaningful problem whether the risk of lung cancer could be reduced by effective prevention and treatment of periodontal disease $[1,4]$. Some previous studies also have reported that the treatment of periodontal disease could reduce the level of inflammatory markers [61-64]. Moreover, Hwang et al. performed a retrospective cohort study including 116,706 periodontal disease patients to explore whether the treatment of periodontal disease could reduce the cancer risks [65]. The result indicated that the treatment of periodontal disease could reduce lung cancer risk in patients with periodontal disease after controlling for age, sex, occupation, type 2 diabetes mellitus, hypertension, and hyperlipidemia $(\mathrm{RR}=0.45,95 \% \mathrm{CI}=0.38-0.54)$ [65]. However, this was an observational study and smoking was not controlled in the analysis, which may affect the validity of results and make it difficult to interpret the results. Therefore, there is an urgent need for large-scale, multicenter clinical studies to explore the prophylactic efficacy of treatment of periodontal disease for lung cancer risk after controlling for multiple confounding factors, especially smoking.
There was considerable heterogeneity in the metaanalysis of the association between periodontal disease and lung cancer risk. The result indicated that the study by Guven et al. contributed relatively substantial heterogeneity [24]. The reason may be that the number of lung cancer cases in the study was lower than that in other cohort studies due to a relatively short follow-up duration, and thus, the limited number of cases may affect the strength of the results. Moreover, the study only controlled for sex and age while the other studies also controlled for other important confounding factors as much as possible such as smoking and alcohol drinking. There was no significant heterogeneity in the meta-analysis of the association between tooth loss and lung cancer risk.

There were several limitations in our meta-analysis. First, the number of included studies was limited, which could affect the implementation of in-depth subgroup analyses. Second, the controlled confounding factors were varied, and the differences may be the potential source of heterogeneity. However, we could not completely control for all important confounding factors and eliminate the heterogeneity because the personal information could not be obtained from these published studies. Third, the exposure ascertainment of periodontal disease was discrepant. Some studies used self-reported measure to ascertain periodontal disease while the other studies used clinical periodontal examination to ascertain periodontal disease. Moreover, there was no uniform amount in the definition of tooth loss.

\section{Conclusions}

Our results indicate that periodontal disease and tooth loss are positively associated with lung cancer risk. Moreover, there is a significantly linear relationship between tooth loss and lung cancer risk, with a monotonically increasing trend. Moreover, subgroup analyses based on different controlled confounding factors including smoking status, amount, and duration also confirm the validity of our results. Further large-scale, well-designed studies are urgently required to adequately control for multiple confounding factors, especially smoking, to explore the association between periodontal disease, tooth loss, and lung cancer risk.

\section{Data Availability}

The data used to support the findings of this study are available from the corresponding author upon request.

\section{Disclosure}

The sponsors had no role in study design, data collection, data analysis, data interpretation, writing of the report, or the decision to submit the paper for publication. The corresponding author had full access to all the data and analyses.

\section{Conflicts of Interest}

The authors declare that there is no conflict of interest regarding the publication of this paper. 


\section{Acknowledgments}

This work was supported by Wenzhou Basic Research Project (Y20180851) by Xi Zhang.

\section{References}

[1] F. Bray, J. Ferlay, I. Soerjomataram, R. L. Siegel, L. A. Torre, and A. Jemal, "Global cancer statistics 2018: GLOBOCAN estimates of incidence and mortality worldwide for 36 cancers in 185 countries," CA: a Cancer Journal for Clinicians, vol. 68, no. 6, pp. 394-424, 2018.

[2] D. R. Youlden, S. M. Cramb, and P. D. Baade, "The international epidemiology of lung cancer: geographical distribution and secular trends," Journal of Thoracic Oncology: Official Publication of the International Association for the Study of Lung Cancer, vol. 3, no. 8, pp. 819-831, 2008.

[3] D. F. Kinane, P. G. Stathopoulou, and P. N. Papapanou, "Periodontal diseases," Nature Reviews Disease Primers, vol. 3, no. 1, p. 17038, 2017.

[4] M. A. Peres, L. M. D. Macpherson, R. J. Weyant et al., "Oral diseases: a global public health challenge," Lancet, vol. 394, no. 10194, pp. 249-260, 2019.

[5] X. T. Zeng, A. P. Deng, C. Li, L. Y. Xia, Y. M. Niu, and W. D. Leng, "Periodontal disease and risk of head and neck cancer: a meta-analysis of observational studies," PloS one, vol. 8, no. 10, p. e79017, 2013.

[6] Q. W. Yao, D. S. Zhou, H. J. Peng, P. Ji, and D. S. Liu, "Association of periodontal disease with oral cancer: a meta-analysis,” Tumor Biology, vol. 35, no. 7, pp. 7073-7077, 2014.

[7] L. Ye, Y. Jiang, W. Liu, and H. Tao, "Correlation between periodontal disease and oral cancer risk: a meta-analysis," Journal of cancer research and therapeutics, vol. 12, Supplement, pp. C237-C240, 2016.

[8] C. C. Abnet, Y. L. Qiao, S. D. Mark, Z. W. Dong, P. R. Taylor, and S. M. Dawsey, "Prospective study of tooth loss and incident esophageal and gastric cancers in China," Cancer causes \& control: CCC, vol. 12, no. 9, pp. 847-854, 2001.

[9] C. C. Abnet, F. Kamangar, S. M. Dawsey et al., "Tooth loss is associated with increased risk of gastric non-cardia adenocarcinoma in a cohort of Finnish smokers," Scandinavian Journal of Gastroenterology, vol. 40, no. 6, pp. 681-687, 2009.

[10] P. Maisonneuve, S. Amar, and A. B. Lowenfels, "Periodontal disease, edentulism, and pancreatic cancer: a meta-analysis," Annals of oncology: official journal of the European Society for Medical Oncology, vol. 28, no. 5, pp. 985-995, 2017.

[11] J.-H. Lee, H. H.-I. Kweon, J.-K. Choi, Y.-T. Kim, and S.H. Choi, "Association between periodontal disease and prostate cancer: results of a 12-year longitudinal cohort study in South Korea," Journal of Cancer, vol. 8, no. 15, pp. 29592965, 2017.

[12] A. Stang, "Critical evaluation of the Newcastle-Ottawa scale for the assessment of the quality of nonrandomized studies in meta-analyses," European Journal of Epidemiology, vol. 25, no. 9, pp. 603-605, 2010.

[13] J. Zhang and K. F. Yu, "What's the relative risk? A method of correcting the odds ratio in cohort studies of common outcomes," JAMA, vol. 280, no. 19, pp. 1690-1691, 1998.

[14] S. Greenland and M. P. Longnecker, "Methods for trend estimation from summarized dose-response data, with applica- tions to meta-analysis," American Journal of Epidemiology, vol. 135, no. 11, pp. 1301-1309, 1992.

[15] N. H. Sun, X. Z. Huang, S. B. Wang et al., "A dose-response meta-analysis reveals an association between vitamin B12and colorectal cancer risk," Public health nutrition, vol. 19, no. 8, pp. 1446-1456, 2016.

[16] V. Bagnardi, A. Zambon, P. Quatto, and G. Corrao, "Flexible Meta-Regression Functions for Modeling Aggregate Dose-Response Data, with an Application to Alcohol and Mortality," American Journal of Epidemiology, vol. 159, no. 11, pp. 1077-1086, 2004.

[17] N. Orsini, R. Li, A. Wolk, P. Khudyakov, and D. Spiegelman, "Meta-analysis for linear and nonlinear dose-response relations: examples, an evaluation of approximations, and software," American Journal of Epidemiology, vol. 175, no. 1, pp. 66-73, 2012.

[18] J. P. Higgins, S. G. Thompson, J. J. Deeks, and D. G. Altman, "Measuring inconsistency in meta-analyses," $\backslash B M J$, vol. 327, no. 7414, pp. 557-560, 2003.

[19] L. M. O’Keeffe, G. Taylor, R. R. Huxley, P. Mitchell, M. Woodward, and S. A. E. Peters, "Smoking as a risk factor for lung cancer in women and men: a systematic review and meta-analysis," BMJ Open, vol. 8, no. 10, p. e021611, 2018.

[20] C. B. Begg and M. Mazumdar, "Operating characteristics of a rank correlation test for publication bias," Biometrics, vol. 50, no. 4, pp. 1088-1101, 1994.

[21] M. Egger, G. D. Smith, M. Schneider, and C. Minder, "Bias in meta-analysis detected by a simple, graphical test," $B M J$, vol. 315, no. 7109, pp. 629-634, 1997.

[22] S. Duval and R. Tweedie, "Trim and fill: a simple funnel-plotbased method of testing and adjusting for publication bias in meta-analysis," Biometrics, vol. 56, no. 2, pp. 455-463, 2000.

[23] H. S. Yoon, W. Wen, J. Long, W. Zheng, W. J. Blot, and Q. Cai, "Association of oral health with lung cancer risk in a lowincome population of African Americans and European Americans in the Southeastern United States," Lung cancer (Amsterdam, Netherlands), vol. 127, pp. 90-95, 2019.

[24] D. C. Güven, Ö. Dizdar, A. C. Akman et al., "Evaluation of cancer risk in patients with periodontal diseases," Turkish Journal of Medical Sciences, vol. 49, no. 3, pp. 826-831, 2019.

[25] Y. Goto and K. Wada, Number of teeth and all-cause and cancer mortality in a Japanese community: the Takayama study, Journal of Epidemiology, 2019.

[26] D. S. Michaud, J. Lu, A. Y. Peacock-Villada et al., "Periodontal disease assessed using clinical dental measurements and cancer risk in the ARIC study," JNCI: Journal of the National Cancer Institute, vol. 110, no. 8, pp. 843-854, 2018.

[27] P. Heikkilä, A. But, T. Sorsa, and J. Haukka, "Periodontitis and cancer mortality: register-based cohort study of 68,273 adults in 10-year follow-up," International Journal of Cancer, vol. 142, no. 11, pp. 2244-2253, 2018.

[28] N. N. Nwizu, J. R. Marshall, K. Moysich et al., "Periodontal disease and incident cancer risk among postmenopausal women: results from the women's health initiative observational cohort," Cancer Epidemiology Biomarkers and Prevention, vol. 26, no. 8, pp. 1255-1265, 2017.

[29] N. A. Chrysanthakopoulos, "Correlation between periodontal disease indices and lung cancer in Greek adults: a case-control study," Experimental oncology, vol. 38, no. 1, pp. 49-53, 2016.

[30] M. Arora, J. Weuve, K. Fall, N. L. Pedersen, and L. A. Mucci, "An exploration of shared genetic risk factors between 
periodontal disease and cancers: a prospective co-twin study," American Journal of Epidemiology, vol. 171, no. 2, pp. 253259, 2010.

[31] D. S. Michaud, Y. Liu, M. Meyer, E. Giovannucci, and K. Joshipura, "Periodontal disease, tooth loss, and cancer risk in male health professionals: a prospective cohort study," The Lancet Oncology, vol. 9, no. 6, pp. 550-558, 2008.

[32] A. Hiraki, K. Matsuo, T. Suzuki, T. Kawase, and K. Tajima, "Teeth loss and risk of cancer at 14 common sites in Japanese," Cancer Epidemiology Biomarkers \& Prevention, vol. 17, no. 5, pp. 1222-1227, 2008.

[33] Y. K. Tu, B. Galobardes, G. D. Smith, P. McCarron, M. Jeffreys, and M. S. Gilthorpe, "Associations between tooth loss and mortality patterns in the Glasgow Alumni Cohort," Heart, vol. 93, no. 9, pp. 1098-1103, 2007.

[34] P. P. Hujoel, M. Drangsholt, C. Spiekerman, and N. S. Weiss, "An Exploration of the Periodontitis-Cancer Association," Annals of epidemiology, vol. 13, no. 5, pp. 312-316, 2003.

[35] B. Blicher, K. Joshipura, and P. Eke, "Validation of selfreported periodontal disease: a systematic review," Journal of Dental Research, vol. 84, no. 10, pp. 881-890, 2016.

[36] H. M. Abbood, J. Hinz, G. Cherukara, and T. V. Macfarlane, "Validity of self-reported periodontal disease: a systematic review and meta-analysis," Journal of Periodontology, vol. 87, no. 12, pp. 1474-1483, 2016.

[37] E. Montero, M. La Rosa, E. Montanya et al., "Validation of self-reported measures of periodontitis in a Spanish population," Journal of Periodontal Research, vol. 55, no. 3, pp. 400-409, 2020.

[38] B. Heaton, N. B. Gordon, R. I. Garcia et al., "A clinical validation of self-reported periodontitis among participants in the Black Women's Health Study," Journal of periodontology, vol. 88, no. 6, pp. 582-592, 2017.

[39] X. Wu, H. Weng, and X. Lin, "Self-reported questionnaire for surveillance of periodontitis in Chinese patients from a prosthodontic clinic: a validation study," Journal of clinical periodontology, vol. 40, no. 6, pp. 616-623, 2013.

[40] Y. Khader, R. Alhabashneh, and F. Alhersh, "Development and validation of a self-reported periodontal disease measure among Jordanians," International Dental Journal, vol. 65, no. 4, pp. 203-210, 2015.

[41] P. de Groot and R. F. Munden, "Lung cancer epidemiology, risk factors, and prevention," Radiologic clinics of North America, vol. 50, no. 5, pp. 863-876, 2012.

[42] Y. A. AlJehani, "Risk factors of periodontal disease: review of the literature," International journal of dentistry, vol. 2014, Article ID 182513, 9 pages, 2014.

[43] L. M. Coussens and Z. Werb, "Inflammation and cancer," Nature, vol. 420, no. 6917, pp. 860-867, 2002.

[44] H. Kuper, H. O. Adami, and D. Trichopoulos, "Infections as a major preventable cause of human cancer," Journal of internal medicine, vol. 248, no. 3, pp. 171-183, 2000.

[45] M. J. Blaser, P. H. Chyou, and A. Nomura, "Age at establishment of Helicobacter pylori infection and gastric carcinoma, gastric ulcer, and duodenal ulcer risk," Cancer Research, vol. 55, no. 3, pp. 562-565, 1995.

[46] E. Shacter and S. A. Weitzman, "Chronic inflammation and cancer," Oncology, vol. 16, no. 2, pp. 217-226, 2002.

[47] N. M. Moutsopoulos and P. N. Madianos, "Low-grade inflammation in chronic infectious diseases: paradigm of periodontal infections," Annals of the New York Academy of Sciences, vol. 1088, no. 1, pp. 251-264, 2006.

[48] K. J. Joshipura, H. C. Wand, A. T. Merchant, and E. B. Rimm, "Periodontal disease and biomarkers related to cardiovascular disease," Journal of dental research, vol. 83, no. 2, pp. 151-155, 2016.

[49] G. E. Salvi, C. E. Brown, K. Fujihashi et al., "Inflammatory mediators of the terminal dentition in adult and early onset periodontitis," Journal of Periodontal Research, vol. 33, no. 4, pp. 212-225, 1998.

[50] C. Hayashi, C. V. Gudino, F. C. Gibson III, and C. A. Genco, "Review: pathogen-induced inflammation at sites distant from oral infection: bacterial persistence and induction of cellspecific innate immune inflammatory pathways," Molecular Oral Microbiology, vol. 25, no. 5, pp. 305-316, 2010.

[51] Y. W. Han and X. Wang, "Mobile microbiome: oral bacteria in extra-oral infections and inflammation," Journal of dental research, vol. 92, no. 6, pp. 485-491, 2013.

[52] M. S. Shiels, R. M. Pfeiffer, A. Hildesheim et al., "Circulating inflammation markers and prospective risk for lung cancer," Journal of the National Cancer Institute, vol. 105, no. 24, pp. 1871-1880, 2013.

[53] D. Brown, A. Zingone, Y. Yu et al., "Relationship between circulating inflammation proteins and lung cancer diagnosis in the National Lung Screening Trial," Cancer Epidemiology Biomarkers \& Prevention, vol. 28, no. 1, pp. 110-118, 2019.

[54] C. L. Meaney, K. A. Mitchell, A. Zingone et al., "Circulating inflammation proteins associated with lung cancer in African Americans," Journal of Thoracic Oncology: Official Publication of the International Association for the Study of Lung Cancer, vol. 14, no. 7, pp. 1192-1203, 2019.

[55] T. Daniluk, G. Tokajuk, D. Cylwik-Rokicka, D. Rozkiewicz, M. L. Zaremba, W. Stokowska et al., "Aerobic and anaerobic bacteria in subgingival and supragingival plaques of adult patients with periodontal disease," Advances in medical sciences, vol. 51, Supplement 1, pp. 81-85, 2006.

[56] F. A. Scannapieco and A. Cantos, "Oral inflammation and infection, and chronic medical diseases: implications for the elderly," Periodontology 2000, vol. 72, no. 1, pp. $153-$ $175,2016$.

[57] L. C. D. Souza, V. Mota, A. Carvalho, R. Corrêa, S. Libério, F. Lopes et al., "Association between pathogens from tracheal aspirate and oral biofilm of patients on mechanical ventilation," Brazilian Oral Research, vol. 31, p. e38, 2017.

[58] T. Kikutani, F. Tamura, H. Tashiro, M. Yoshida, K. Konishi, and R. Hamada, "Relationship between oral bacteria count and pneumonia onset in elderly nursing home residents," Geriatrics \& Gerontology International, vol. 15, no. 4, pp. 417-421, 2015.

[59] D. R. Brenner, J. R. McLaughlin, and R. J. Hung, "Previous lung diseases and lung cancer risk: a systematic review and meta-analysis," PloS one, vol. 6, no. 3, p. e17479, 2011.

[60] A. Cassidy, J. P. Myles, M. van Tongeren et al., "The LLP risk model: an individual risk prediction model for lung cancer," British journal of cancer, vol. 98, no. 2, pp. 270-276, 2008.

[61] J. R. Elter, A. L. Hinderliter, S. Offenbacher et al., "The effects of periodontal therapy on vascular endothelial function: a pilot trial," American Heart Journal, vol. 151, no. 1, p. 47, 2006.

[62] K. Mattila, M. Vesanen, V. Valtonen et al., "Effect of treating periodontitis on C-reactive protein levels: a pilot study," BMC Infectious Diseases, vol. 2, no. 1, p. 30, 2002. 
[63] F. D’Aiuto, M. Parkar, G. Andreou et al., "Periodontitis and systemic inflammation: control of the local infection is associated with a reduction in serum inflammatory markers," Journal of Dental Research, vol. 83, no. 2, pp. 156-160, 2016.

[64] Y. Iwamoto, F. Nishimura, Y. Soga et al., "Antimicrobial periodontal treatment decreases serum C-reactive protein, tumor necrosis factor-alpha, but not adiponectin levels in patients with chronic periodontitis," Journal of Periodontology, vol. 74, no. 8, pp. 1231-1236, 2003.

[65] I.-M. Hwang, L.-M. Sun, C.-L. Lin, C.-F. Lee, and C.-H. Kao, "Periodontal disease with treatment reduces subsequent cancer risks," QJM: monthly journal of the Association of Physicians, vol. 107, no. 10, pp. 805-812, 2014. 